\title{
Prolonged Intraoperative Cardiac Arrest in a Young Patient with Successful Precordial Thump
}

\author{
Authors: \\ Sitelnissa Saeed Ahmed, ${ }^{*}$ Gamal Abdalla Mohamed Ejaimi, ${ }^{2}$ Areeg \\ Izzeldin Ahmed Yousif 3 \\ 1. Intensive Care, Aseer Central Hospital, Abha, Saudi Arabia \\ 2. Department of Anesthesia and Intensive Care, Sabah Al-Salem, Kuwait \\ 3. Department of Anesthesia and Intensive Care, Ahmed Gasim Hospital Heart \\ Surgery and Kidney Transplant Center, Khartoum North, Sudan \\ *Correspondence to gamalejaimi@hotmail.com
}

Disclosure: The authors have declared no conflicts of interest.

Received: $\quad 02.02 .20$

Accepted: $\quad 30.03 .20$

Keywords: $\quad$ Anaesthesia, cardiac arrest, cardiopulmonary resuscitation (CPR), intraoperative, postresuscitation care, pulmonary embolism (PE).

Citation:

EMJ. 2020;DOI/10.33590/emj/20-00024.

\section{Abstract}

Cardiac arrest during surgery is rare but is one of the most dreaded complications. Precordial thump (PT) had been used for a long time, but in the present day it has become obsolete. In regard to the witnessed onset of asystole, there is insufficient evidence to recommend for or against the use of the PT. This case report is of a 17-year-old male who presented to hospital with a congenital haemangioma on the right calf. He had no other significant medical conditions and was on no other medications. The patient history, clinical examination, and investigations were normal. He had undergone an operation 3 weeks previously where a section of his haemangioma was excised, and an appointment was made for excision of the remaining haemangioma. Anaesthesia induction and endotracheal intubation were smooth and uneventful. Following lifting and exsanguination of the patient's leg by Esmarch bandage, he developed ventricular fibrillation and arrested with asystole. Cardiopulmonary resuscitation was performed, with no good response, for approximately 50 minutes. Lastly, a PT was performed, and the patient's heart rate immediately returned. The operation was postponed. Postresuscitation care was conducted in an intensive care unit. The patient was later discharged without complications.

\section{INTRODUCTION}

Cardiac arrest during surgery is rare but is one of the most dreaded complications, particularly when there is no obvious underlying cause or risk. Some studies conducted between 2001 and 2012 quoted the incidence of intraoperative cardiac arrest (ICA) to be 1.10-7.22 per 10,000 surgeries..$^{1-4}$ Cardiac arrests related to anaesthesia are classified as avoidable but are thought to have a higher survival rate compared to other possible causes of cardiac arrest in the operating room. Anaesthesia-attributable cardiac arrests are related to airway management and medication administration, which are important considerations in prevention strategies. ${ }^{2,3}$ Post-cardiac arrest care using a highquality integrated multisystem of support and management could significantly affect patient outcome. Post-cardiac arrest care should be implemented according to the 2015 American Heart Association Guidelines Update for Cardiopulmonary Resuscitation and Emergency Cardiovascular Care (AHA CPR ECC) ${ }^{5}$ 
A 17-year-old male presented to the hospital with a congenital haemangioma on the right calf, measuring approximately $9 \times 10 \mathrm{~cm}$. His body weight was $60 \mathrm{~kg}$. The patient had no other complaints and no relevant medical history. $\mathrm{He}$ had a heart rate of $80 /$ minute, respiratory rate of 17/minute, blood pressure of 120/70, and oxygen saturation $\left(\mathrm{SpO}_{2}\right)$ of $98 \%$. Clinical examination of the cardiovascular, respiratory, and nervous systems was normal. He had undergone an operation 3 weeks previously where a section of his haemangioma was excised, and an appointment was made for excision of the remaining haemangioma. The investigations, including complete blood count, random blood sugar, liver function test, urea, creatinine, and electrolytes, were all within normal limits. The patient fasted for 8 hours prior to the operation, as per hospital protocol, with intravenous fluids maintenance. The patient was connected to standard monitoring as per the American Society of Anesthesiologists (ASA) guidelines. ${ }^{6}$ After preoxygenation with $100 \% \mathrm{O}_{2}$, anaesthesia was induced with $100 \mu \mathrm{g}$ fentanyl, $130 \mathrm{mg}$ propofol, and $35 \mathrm{mg}$ rocuronium. The tracheal intubation was performed uneventfully. Anaesthesia was maintained with $50 \% \mathrm{O}_{2}$ in $\mathrm{N}_{2} \mathrm{O}$, with an isoflurane minimum anaesthetic concentration of $1.6 \%$. All patient and ventilator parameters were normal. The patient's leg was lifted by the surgeon when applying the Esmarch bandage. Immediately following exsanguinations and compression over both the calf and the site of the previous operation, the patient developed ventricular fibrillation (VF) and became hypotensive and arrested in asystole. Effective cardiopulmonary resuscitation (CPR) was started with 100\% fraction of inspired oxygen, bag valve mask ventilation, and cardiac compressions. Checking of the equipment, machines, and breathing system, as well as verification of the endotracheal tube, were completed with no defects reported. Hypoglycaemia was ruled out. Adrenaline was given intravenously at $1 \mathrm{mg}$ every 3-5 minutes, up to a total dose of $10 \mathrm{mg}$. Intravenous 300 and 150 $\mathrm{mg}$ amiodarone were administered. There was no good response for approximately 50 minutes.

The reversible causes of cardiac arrest ('four Hs and four Ts') were checked simultaneously alongside the ongoing effective CPR. Lastly, a precordial thump (PT) was delivered by a senior anaesthetist, and the patient's heart rate immediately returned. With a firmly clenched right fist, at approximately $20 \mathrm{~cm}$ above the patient, a strike was given on the inferior third of the patient sternum. The ECG showed a narrow QRS complex tachycardia which then changed to a sinus rhythm. The end-tidal $\mathrm{CO}_{2}$ on the capnograph was raised immediately. The operation was postponed. The patient was stabilised and transferred to an intensive care unit (ICU). Monitoring was continued, and an arterial line was inserted for invasive blood pressure and repeated arterial blood gases sampling. Synchronised intermittent mechanical ventilation mode was used. Four hours later, the patient recovered his spontaneous respiration and his level of consciousness began to improve. Two hours after this he was extubated safely.

A pulmonary embolism (PE) that migrated from the location of the previous surgery was thought to be the most likely diagnosis. He was kept in the ICU on 5,000 $U$ heparin intravenous bolus, followed by a continuous infusion of 1,200 U/hour until the following day. On Day 2, he was discharged to the intermediate care unit where he stayed for 2 days. The cardiology, haematology, respiratory, and neurology evaluations, examinations, and investigations were completed and revealed no abnormality, apart from a small thrombus at the site of previous surgery, identified by a doppler ultrasound.

\section{DISCUSSION}

Intraoperative blood loss, as indicated by the volume of transfusion, was the most important predictor of ICA. ASA status and functional status were other important risk factors.-4 Cardiac arrests entirely related to anaesthesia are classified as avoidable and are thought to have a higher survival rate compared to other possible causes of cardiac arrest in the operating room. The study by Braz et al. ${ }^{3}$ concluded that all anaesthesia-attributable cardiac arrests were related to airway management and medication administration, which are both important considerations in prevention strategies. ${ }^{2,3}$ During surgery, the context of positioning and specific procedures may have a particular effect on cardiac arrest. However, a proper understanding of the mechanisms and management options related to these events 
would certainly assist in minimising adverse outcomes. ${ }^{7}$ The outcome of cardiac arrest during anaesthesia is generally good, with most patients leaving the hospital alive and seemingly well. A complete systematic assessment of the patient, equipment, and drugs should be completed during an ICA, even if the cause of the cardiac arrest is already hypothesised. ${ }^{8}$ Cardiac arrests during nonregular working hours tend to have worse outcomes, which may indicate that the availability of human resources influences survival. ${ }^{4}$

Deep venous thrombosis, in most reported cases, involves the lower extremities. PE can occur in young patients with unknown risk factors and be without anticipation. Schonauer et al. ${ }^{9}$ reported a large $\mathrm{PE}$ in a 23 -year-old woman following bilateral breast augmentation. The authors recommended more restrictive risk assessment scores, such as the Caprini score, because of a possible higher risk of complications secondary to anticoagulant treatment such as bleeding and haematoma. ${ }^{9}$ Anticoagulation is the standard for management of PE. The addition of aggressive treatment such as thrombolysis or surgical embolectomy may be needed when the clot burden or clinical presentation warrant such measures. Improvement in forward flow and a reduction in right ventricle afterload, to avoid right ventricular failure, is the goal of therapy of large PE. Reducing the degree of occlusion in the pulmonary vasculature and minimising the humoral vasoconstrictors that emanate from intravascular thrombi (mainly serotonin and histamine, produced by neutrophils, endothelium, and platelets), results in a fall in pulmonary vascular resistance and pulmonary artery pressure. ${ }^{10}$

PT had been used for a long time but in the present day it has become obsolete. For unwitnessed out-of-hospital cardiac arrest, the PT should not be used (Class III, Levels of Evidence C). It is not recommended as first-line treatment for VF, but is used in cases in which there is no defibrillator available. It is not effective in terminating VF and may cause rhythm deterioration. For witnessed onset of asystole, there is insufficient evidence to recommend for or against the use of the PT. ${ }^{11,12}$ Few studies have described the value of PT as first-line treatment to monitored out-of-hospital cardiac arrest as a result of VF and pulseless ventricular tachycardia. ${ }^{13}$ A study by Madias et al. ${ }^{14}$ recommended PT for asystole. ${ }^{14}$ A serial PT was successfully used in a 28-day-old newborn baby who presented with a refractory supraventricular tachycardia and cardiovascular collapse after amiodarone administration. ${ }^{15}$ Post-cardiac arrest care using a high-quality integrated multisystem of support and management could significantly affect patient outcome. Post-cardiac arrest should be implemented following the 2015 AHA CPR ECC. ${ }^{5}$ In the patients undergoing valve surgery, measuring postoperative high-sensitivity troponin $T$ is recommended because its elevation is associated with a high risk of postoperative, sudden cardiac arrest. ${ }^{16}$ The most likely cause of cardiac arrest in this patient was migration of an embolism from the site of previous surgery. This diagnosis was made based on clinical judgement, as well as the finding of the small emboli on the doppler ultrasound. PE was not ruled out during the cardiac arrest by echocardiogram due to its unavailability at the time. In ICU, the patient was stable, so no further investigations were requested by the cardiology or pulmonology staff.

\section{CONCLUSION}

ICA in a young, healthy patient is a feared event. However, anaesthetists should have the capability to deal with such situation. Early recognition, initiation of effective CPR, and established postresuscitation care allow the maximum potential for a good outcome. PT may still have a role in witnessed cardiac asystole.

\section{References}


from a Brazilian teaching hospital. Br J Anaesth. 2006:96(5):569-75

4. Sprung $\mathrm{J}$ et al. Predictors of surviva following cardiac arrest in patients undergoing noncardiac surgery: a study of 518,294 patients at a tertiary referral center. Anesthesiology. 2003:99(2):259-69

5. Soar J et al. Part 4: advanced life support: 2015 international consensus on cardiopulmonary resuscitation and emergency cardiovascular care science with treatment recommendations. Resuscitation. 2015:95:e71-120.

6 Chilkoti G et al. Technological advances in perioperative monitoring: current concepts and clinical perspectives. Anaesthesiol Clin Pharmacol. 2015:31(1):14

7. Chowdhury T et al. Cardiac emergencies in neurosurgical patients. Biomed Res Int. 2015;2015:751320

8. Runciman $\mathrm{W}$ et al. Crisis management during anaesthesia: cardiac arrest. Qual Saf Health Care. 2005:14(3):e14

9. Schonauer $\mathrm{F}$ et al. Episode of massive oulmonary embolism after bilateral breast augmentation. Case Reports Plast Surg Hand Surg. 2015:2(3-4):80-3

10. Cooper JM, Beckman JA. Massive pulmonary embolism: a remarkable case and review of treatment Vasc Med 2002:7(3):181-5

11. Cave DM et al. Part 7: CPR techniques and devices: 2010 American

Heart Association guidelines for cardiopulmonary resuscitation and emergency cardiovascular care. Circulation. 2010:122(18 Suppl 3):5720-8.

12. Smith J, Judge B. BET 1: effectiveness of the precordial thump in restoring heart rhythm following out-ofhospital cardiac arrest. Emerg Med $J$. 2016:33(5):366-7.

13. Nehme Z et al. Treatment of monitored out-of-hospital ventricular fibrillation and pulseless ventricular tachycardia utilising the precordial thump. Resuscitation. 2013;84(12):1691-6.

14. Madias C et al. Precordial thump for cardiac arrest is effective for asystole but not for ventricular fibrillation. Heart Rhythm. 2009:6(10):1495-500

15. Jan SL et al. Precordial thump in a newborn with refractory supraventricular tachycardia and cardiovascular collapse after amiodarone administration. Eur J Emerg Med. 2012:19(2):128-9

16. Duchnowski P et al. Postoperative high sensitivity troponin $T$ as a predictor of sudden cardiac arrest in patients undergoing cardiac surgery. Cardiol J. 2019:26(6):777-81 